\title{
Le labyrinthe de l'idéalisme: Scepticisme, réalisme et idéalisme dans l'élaboration de la « Doctrine de la science » de 1794
}

Isabelle Thomas-Fogiel

\section{(2) OpenEdition \\ Journals}

Édition électronique

URL : http://journals.openedition.org/ref/701

ISSN : 2258-014X

\section{Éditeur}

EuroPhilosophie Editions

\section{Référence électronique}

Isabelle Thomas-Fogiel, « Le labyrinthe de l'idéalisme: Scepticisme, réalisme et idéalisme dans

l'élaboration de la « Doctrine de la science » de 1794 », Revista de Estud(i)os sobre Fichte [En línea], 13 |

2017, Publicado el 01 marzo 2017, consultado el 08 septiembre 2020. URL : http://

journals.openedition.org/ref/701

Ce document a été généré automatiquement le 8 septembre 2020

(c) EuroPhilosophie 


\title{
Le labyrinthe de l'idéalisme: Scepticisme, réalisme et idéalisme dans l'élaboration de la « Doctrine de la science » de 1794
}

\author{
Isabelle Thomas-Fogiel
}

A Isabelle Berner

\section{Introduction}

1 Si l'on examine la relation de Fichte à Kant à la lumière de la question de l'idéalisme, la différence entre les deux auteurs se révèle saisissante. En effet, si Kant est le premier philosophe à se dire "idéaliste ", Fichte est le premier à faire de la question de l'idéalisme le cœur et le moteur de toute démarche philosophique, anticipant la célèbre formule de Hegel : «toute vraie philosophie est idéaliste » ou n'est pas philosophie ${ }^{1}$. Dès lors Fichte, plus encore que Kant, est à l'origine de cette formidable lame de fond que fut "l'idéalisme allemand", que Dieter Henrich déterminera comme une "constellation conceptuelle», soit l'adoption, à une même époque, de principes structurels identiques, de gestes cognitifs analogues qui se cristallisent, à un moment donné, autour d'une catégorie fédératrice. C'est bien Fichte et non Kant qui a reconfiguré l'espace philosophique en conférant à la notion «d'idéalisme » une centralité, historiquement sans précédent.

2 De fait, dès la première doctrine de la science en 1794 (Grundlage der gesamten Wissenschaftslehre), l'antinomie entre réalisme et idéalisme est à ce point structurante qu'elle rythme l'ensemble des développements puisque sa tentative de résolution conditionne la progression de toutes les démonstrations. La manière d'argumenter de Fichte y est la suivante : une contradiction est donnée (sur la base des trois premiers principes initialement énoncés); cette contradiction est levée par une synthèse ou composition des termes; mais cette synthèse produit à son tour une nouvelle 
contradiction. La Doctrine de la science, nous dit Fichte "procède toujours en introduisant des moyens termes entre les termes opposés ; mais par-là la contradiction n'est pas résolue mais simplement déplacée $~^{2}$. A chaque étape de la synthèse apparaîtront les positions idéalistes et réalistes correspondant au niveau atteint. C'est cette centralité de l'antinomie qui explique la subtilité, quasi byzantine, des distinctions établies par Fichte tout au long de la Grundlage (idéalismes et réalismes qualitatif, quantitatif, abstrait). La recension de ces différentes figures aporétiques permettra l'accès final à la vérité.

3 Certes, à cette affirmation selon laquelle c'est Fichte qui a fait de l'idéalisme le concept central de toute philosophie future, il serait loisible d'objecter que Kant est, quand même, le premier philosophe à se dire explicitement « idéaliste ». De fait, si le terme fut introduit en 1702 par Leibniz ${ }^{3}$ et repris en 1719 par Wolff ${ }^{4}$, il n'en demeure pas moins que toutes ces occurrences avant Kant attribuent le terme rétrospectivement à un auteur qui ne l'utilisait pas pour lui-même. Ainsi Leibniz l'institue pour qualifier la théorie des idées de Platon, et donc sans consentement de l'intéressé. De même, Wolff cite-t-il, comme exemple concret d'idéaliste, Berkeley, alors même que ce dernier n'a nulle part revendiqué ce terme, mais se voulait «immatérialiste ». Enfin Diderot, en 1749, enrôle dans le camp de l'idéalisme Berkeley et Malebranche (auxquels il adjoint de manière surprenante Condillac) $)^{5}$, tous philosophes qui ne se définirent jamais comme «idéalistes". Ainsi Kant est bien le premier philosophe à se vouloir explicitement "idéaliste», et pourrait donc se voir attribuer la paternité de cette constellation conceptuelle que fut « l'idéalisme allemand».

4 Néanmoins, nous pouvons répondre à cette objection en remarquant que Kant n'a jamais vraiment considéré la question de l'idéalisme comme décisive. En effet, l'intervention de Kant, dans le débat sur la juste signification de l'idéalisme, a été induite de l'extérieur. Il convient, dès lors, de ne pas céder à l'illusion rétrospective et croire que Kant a accordé, d'emblée, une signification centrale à la question de sa situation face à d'éventuels idéalistes plus anciens. Ce n'est qu'après la recension de Garve et Feder ${ }^{6}$ (qui l'associe à Berkeley et le taxe " d'idéalisme transcendant »), qu'il prend conscience d'une possible équivoque et y répond d'abord en 1783, dans les Prolégomènes ${ }^{7}$, puis ensuite, en 1787 , en modifiant la seconde édition.

Or, dans la première édition, les mentions de l'idéalisme non transcendantal sont relativement peu nombreuses: on en trouve que trois occurrences ${ }^{8}$, dont une seule vraiment significative et Berkeley n'y est jamais nommé. Bref, la querelle entre l'idéalisme et le réalisme n'est pas un enjeu majeur dans l'économie de la critique, car même si l'intervention de Garve et Feder obligea Kant à supprimer le quatrième paralogisme et à écrire, dans la seconde édition, une "réfutation de l'idéalisme ", il n'en demeure pas moins que cette question n'a ni infléchi ni transformé le cours général de la Critique.

Ces considérations préalables nous autorisent à faire surgir une différence entre Kant et Fichte, quant à la question de l'idéalisme. Pour Kant, la définition et la réfutation de l'idéalisme non transcendantal est présente, certes, mais non centrale. Il s'agit, en fait, d'une mise au point mais non de la pièce maitresse de la démonstration. Pour Fichte, c'est l'inverse, puisque toute l'argumentation de son premier grand texte de philosophie, la Grundlage de 94, repose sur la caractérisation progressive des différents types d'idéalismes et de réalismes, dont le dépassement sera la condition d'accès à la 
véritable philosophie. Ainsi Kant ne se soucie que tardivement des autres formes d'idéalisme, alors que Fichte part des différents idéalismes.

7 De ce constat surgit une question : pourquoi Fichte, en 94, fait-il de l'antinomie du réalisme et de l'idéalisme le point essentiel de son élaboration philosophique ? En quoi, l'exacte détermination du terme «idéaliste » était, après Kant, à ce point nécessaire ? Ou pour le dire encore autrement : pourquoi l'idéalisme kantien (idéalisme "critique », «formel» ou «transcendantal», selon les trois termes utilisés par Kant pour caractériser sa doctrine) s'est-il révélé à ce point insuffisant, aux yeux de Fichte, qu'il lui a fallu, dans la Grundlage, refonder l'ensemble de la philosophie à partir d'une plongée dans le «labyrinthe de l'idéalisme » et procéder à une entière redéfinition de la notion?

Pour répondre à cette question, je commencerai par remonter au problème à l'origine de la doctrine de la science. Pourquoi Fichte en 1793 juge-t-il la Critique insuffisante, l'idéalisme kantien "inachevé " ${ }^{9}$, et se met-il à réclamer une doctrine de la science, qui, notons-le au passage, n'est ni une doctrine du sujet, ni une doctrine de la liberté, ni une doctrine de l'absolu mais bel et bien une doctrine de la science et le restera jusqu'en 1814. Ensuite, j'analyserai la signification au sein de la Grundlage des différents réalismes et idéalismes (qualitatif, quantitatif et abstrait). Ce qui me permettra de montrer que l'idéalisme fichtéen n'est pas lié à une quelconque "philosophie du sujet ", mais à la question de l'idéalité d'un énoncé et s'est noué à partir du problème de l'énonciation du discours du philosophe. Sera idéaliste transcendantal celui qui peut rendre compte de la possibilité de l'acte d'énonciation des énoncés philosophiques. Je montrerai, pour finir, que la philosophie contemporaine, qui se caractérise par sa critique virulente de l'idéalisme, commet un lourd contresens qui consiste d'une part à rattacher le concept d'idéalisme à la notion de subjectivité quand il se rattache depuis sa naissance à la notion d'idéalité, et d'autre part à le penser à partir de la catégorie de réalité ou d'existence de la chose, là où il est d'abord question de la validité du sens.

\section{I) Archéologie de la Grundlage : l'importance de l'offensive sceptique}

\section{I.1. Raisons qui ont motivé Fichte à « tout rebâtir».}

Avant que d'analyser la signification et l'importance de l'antinomie de l'idéalisme et du réalisme dans la Grundlage, il est nécessaire de revenir aux motivations qui engagèrent Fichte à reconstruire le criticisme et à élaborer sa première doctrine de la science. Fichte nous a indiqué très précisément la cause qui a rendu, à ses yeux, la reconstruction nécessaire, à savoir l'attaque sceptique contre la critique. Dans une lettre à Stephani de décembre 1793, il écrit : “ Avez-vous lu Enésidème ? Il m’a bouleversé un certain temps, a renversé en moi Reinhold, m'a rendu Kant suspect [...]. Il fallait rebâtir, c'est ce que je fais avec ardeur depuis six semaines"; et il précise peu de temps après, dans une lettre de janvier 1794 : "Enésidème a définitivement assis ma conviction que dans son état actuel la philosophie n'était pas une science "10. C'est donc l'offensive du scepticisme post-kantien qui contraint Fichte à réviser sa relation à Kant et l'engage selon ses termes "à tout rebâtir ». C'est ce que corrobore le fait que le même terme fort de "bouleversement" reviendra sous sa plume à propos d'un autre sceptique, Maïmon ${ }^{11}$. Aussi n'est-il nullement excessif de prétendre que la question qui présida à 
l'élaboration de la doctrine de la science, et en commanda la structure, naquit sous l'impulsion du scepticisme post-kantien, incarné par Enésidème et Maimon.

\section{I.2. Le scepticisme avant et après Kant.}

10 Mais pour bien comprendre ce «bouleversement », ressenti par Fichte, il est crucial de remarquer que le scepticisme après Kant a changé de signification. Le scepticisme antérieur à la Critique porte sur le lien entre la connaissance et l'être, autrement dit sur le rapport du sujet à l'objet. Ce que Hume conteste est le passage de la relation causale dans notre esprit à son objectivité dans les choses. Le scepticisme de Hume, auquel répond la Critique, est relatif à la possibilité du rapport réglé entre sujet et objet. Ce qui fait le caractère spécifique du scepticisme avant Kant est sa contestation explicite du rapport entre le cours de nos représentations et l'ordre des choses.

11 Or, après Kant, cette question disparait des réfutations ou des lectures sceptiques de la Critique. La question “qu'est ce qui fait l'objectivité de nos représentations?" n'est, paradoxalement, plus un enjeu important. En effet, la critique directrice d'Enésidème, comme de Maimon, concernera la relation de Kant à ses propres propositions. Quel est le statut du discours du philosophe? Quels sont les modes de savoir qu'il doit mettre en œuvre pour expliquer ce qu'il explique, à savoir la représentation conçue comme relation du sujet à l'objet? Le philosophe Kant prétend retracer le fonctionnement de la conscience en tant qu'elle est connaissance d'objet, mais ne nous dit rien des actes ou procédures par lesquels il accède à la connaissance de ce fonctionnement, c'est-à-dire à la connaissance du sujet et de ses structures.

Il s'agit donc, pour ces sceptiques, d'interroger la prétention à la validité du discours de Kant. La remise en cause de cette prétention à la validité prend toujours, chez eux, la même forme : la philosophie de Kant se réfute d'elle-même. Kant ne peut justifier la vérité de ses propres thèses qu'en présupposant des principes que ces thèses mêmes ont pour but de récuser. Sa notion de " validité » est donc auto-contradictoire. Telle est ce que résume l'alternative finale à laquelle Enésidème tente de réduire le criticisme :

13 1) ou bien ce que dit la Critique est vrai et alors pour qu'une connaissance soit vraie, il faut qu'il y ait une intuition et un concept. Or, l'énumération des conditions de possibilité de la connaissance ne satisfait pas à ce critère de vérité, puisque ces conditions ne sont pas représentables par intuition et concepts. Par suite, si ce que dit la Critique de la raison pure est vrai, alors la Critique de la raison pure est fausse ;

2) ou bien, il existe un mode de vérité autre que la liaison d'un concept et d'une intuition. En ce cas, Kant a tort de dire que la seule vérité est celle des jugements mathématiques et physiques. Par suite, là encore, la Critique de la raison pure est fausse ${ }^{12}$.

\section{I.3. La véritable question de la doctrine de la science.}

Nous pouvons, à la lumière de cette restitution, comprendre l'onde de choc que provoqua l'offensive sceptique sur Fichte encore kantien (ou plus exactement reinholdien) avant sa lecture d'Enésidème. La question à laquelle doit s'atteler la doctrine de la science devient moins celle de la représentation que celle de la réflexion. Comment Kant peut-il dire ce qu'il dit? Kant trace la cartographie de l'esprit humain (douze catégorie, deux intuitions, etc.), mais ne nous dit rien de la manière dont il peut accéder à ce savoir. « D'où le sait-il ? » martèlera Fichte, comme un refrain, dans l'essai 
d'une nouvelle présentation de la doctrine de la science. Le problème que Fichte affronte n'est donc plus seulement de savoir comment le sujet connaît mais de déterminer comment le philosophe peut décrire la manière dont le sujet connaît (« d'où le saitil ?»).

C'est cette question à l'origine de la doctrine de la science qui permet de comprendre l'importance et la complexification de l'opposition entre idéalisme et réalisme au sein de la Grundlage. Kant, nous l'avons dit, doit expliquer la relation sujet/objet, c'est-à-dire ce que l'on pourrait nommer la structure de l'intentionnalité ou de la représentation. Comment nos jugements ou nos propositions de sujet peuvent-ils dire quelque chose de l'objet? Dans ce cadre, la bataille philosophique est relativement balisée : le sceptique de type humien niera la concordance entre la marche de la nature ${ }^{13}$ et nos principes rationnels (principe de raison suffisante, de cause et d'effet, de connexion sous une loi). Contre lui, Kant cherchera à établir cette concordance. Il le fera sans renouer avec le réalisme classique qui prétend que c'est l'objet (le cours de la nature) qui nous dicte, comme "le maitre à l'écolier ", ce que nous devons en dire, mais sans épouser, pour autant, la position idéaliste qui abandonne l'objet aux monde des apparences et n'entend plus statuer que sur le monde intelligible, ce qui est la définition, par Kant, de "l'idéalisme transcendant " ${ }^{14}$. Les positions sceptiques, idéalistes et réalistes se distribuent ici autour d'un même axe puisque ce qu'il s'agit d'expliquer est la représentation adéquate par le sujet d'un objet extérieur (relation sujet/objet).

Mais l'attaque des sceptiques post-kantiens a introduit dans le débat un autre terme ou un niveau supplémentaire. Il y a la relation sujet/objet qui doit être expliquée, mais aussi la possibilité pour le philosophe de pouvoir dire cette relation sujet/objet sans contradiction. Nous avons donc deux instances différentes : 1) le sujet intentionnel ou représentant. Ce premier sujet est le sujet en tant qu'il est essentiellement relation à l'objet, ouverture au monde. Il s'agit du sujet engagé, par exemple dans une perception et qui voit le «fer attiré par l'aimant ». C'est ce sujet que Kant explique dans la Critique de la raison pure, en montrant comment il parvient à rejoindre le monde.

Fichte est confronté à une deuxième instance, à savoir le sujet qui écrit la Critique de la raison pure, c'est-à-dire le philosophe qui dit la structure de la représentation. Il s'agit ici d'un sujet 2 qui explique le sujet 1 . Il y a donc bien deux instances: le sujet intentionnel en tant qu'il est pris dans sa relation à l'objet, et le sujet philosophique qui doit expliquer le sujet intentionnel, et devient par là le sujet de la réflexion. En effet, il n'est plus un sujet qui représente un objet qui n'est pas lui (relation sujet-objet), mais une instance qui étudie le sujet représentant (relation sujet/sujet).

19 Or Fichte est le premier, dans l'histoire de la philosophie à thématiser frontalement cette structure duale ${ }^{15}$. C'est ce jeu entre représentation (sujet intentionnel) et réflexion (le philosophe, qui parle du sujet intentionnel) qui contraindra Fichte à complexifier considérablement l'antinomie entre réalisme et idéalisme et, par-là, à promouvoir une définition nouvelle de l'idéalisme. C'est cette complexification du dispositif qu'il nous faut maintenant étudier au sein de la Grundlage. 


\section{II) L'antinomie du réalisme et de l'idéalisme dans la Grundlage et la signification finale de l'idéalisme fichtéen.}

20 Dans la Grundlage, la structure intentionnelle, comme représentation, se dira par la notion de "relation acte-passivité "(Wechsel-Tun und Leiden); la structure réflexive s'exprimera par la notion « d'activité indépendante » (unabhangige Tätigkeit). Ce sont les deux notions structurantes de la Grundlage ${ }^{16}$. Leur composition (comme synthèse de la représentation et de la réflexion) conduira à la mise en place de ce que Fichte appellera "l'idéalisme achevé établi par la doctrine de la science " ${ }^{17}$. Ces deux notions que la Grundlage doit composer (dans la fameuse synthèse de la déterminabilité de la deuxième partie ${ }^{18}$ ) sont la formalisation de la difficulté rencontrée par Fichte en 1793, à savoir : expliquer non seulement la relation du moi et du non moi (représentation ou relation acte-passivité) mais également la possibilité de dire cette relation (réflexion comme activité indépendante).

Or, les différentes figures de réalisme et d'idéalisme, analysées dans la Grundlage, seront les synthèses erronées des deux séries qui doivent être "composées ». Chacune de ces figures (qualitative, quantitative et abstraite) se situe à un degré différent d'abstraction, où l'on ira du moins abstrait (idéalisme et réalisme qualitatif) au niveau le plus abstrait (idéalisme et réalisme abstrait) ${ }^{19}$. Analysons les différents niveaux de cette antinomie entre réalisme et idéalisme, ce qui nous permettra, par opposition, de faire surgir la juste définition de «l'idéalisme achevé » de Fichte.

\section{II.1. Réalisme et idéalisme qualitatif.}

Le premier niveau intervient assez tôt ${ }^{20}$ dans la Grundlage et peut-être incarné par Spinoza et Leibniz (en fait, Fichte y parle de "quelques leibniziens»). On peut caractériser la nature du geste du réaliste qualitatif de la manière suivante : il s'attache exclusivement à la relation sujet/objet. Son problème n'est donc pas, comme dans la Grundlage, de combiner la relation acte/passivité et l'activité indépendante, mais de partir de la scission sujet/objet et de tenter de la résorber. Le réaliste n'a pas encore atteint le niveau de la thématisation explicite de l'activité indépendante comme réflexion ${ }^{21}$. Cette activité indépendante ou réflexion apparaîtra subrepticement, comme un problème, une ombre portée, une aporie engendrée par la position. Pour penser la seule relation acte-passivité, le réaliste qualitatif applique le principe de causalité. Le sujet devient alors effet de l'objet, pure image d'une instance extérieure qui le conditionne. Fichte écrit « Le moi est purement passif ; les degrés de négation sont sans doute bien posés, mais pour un être intelligent extérieur au moi, qui observerait et jugerait, d'après la règle de détermination, le moi et le non-moi dans cette action [...]. Dans le cas présent le moi serait limité mais ne serait pas conscient de sa limitation ${ }^{22}$. En fait, le réaliste, définissant le sujet représentant comme pur reflet ou simple effet d'une instance extérieure, ne peut expliquer le savoir qu'il a de cette limitation. Le réaliste parle toujours du point de vue "d'un être intelligent extérieur au moi », ce que Putnam appellera bien plus tard « le point de vue de nulle part ou le point de vue de Dieu ». Ce que le réalisme ne parvient pas à expliquer c'est l'acte même du philosophe qui ne peut se penser à son tour et sans contradiction comme absolu reflet. Le réalisme, ici envisagé par Fichte, peut être illustré par le réalisme empirique, qui considère 
l'esprit comme une table rase reflétant un réel extérieur. Mais il peut également renvoyer au spinozisme qui explique l'esprit fini (le mode) comme émanation d'une instance absolue (Dieu ou la nature).

L'idéalisme qualititatif commet la faute symétrique inverse. Le réaliste ne parvenait pas à expliquer la possibilité de sa propre réflexion, son activité de philosophe. Il faisait surgir, comme malgré lui, «l'activité indépendante», qui contredisait performativement l'ensemble de son dispositif ${ }^{23}$. En revanche, l'idéalisme quantitatif ne parvient pas à expliquer la représentation comme passivité ou limitation de l'activité du moi. Comme le réaliste, l'idéaliste qualitatif part de la relation sujet-objet. Mais plutôt que de poser l'objet comme premier, il pose le sujet et utilise pour penser la relation entre les deux termes, la catégorie de substantialité et non plus de causalité. L'objet (le non-moi, la passivité) ne sera rien d'autre que l'accident d'un moi-substance. L'objet sera dès lors pensé comme une "activité diminuée » par rapport à l'activité du moi. Une certaine forme de leibnizianisme peut illustrer plus concrètement ce développement de Fichte. Ainsi pour Leibniz, la passivité de la représentation doit être expliquée à partir de la seule activité. L'imagination, par exemple, sera pensée comme activité obscurcie par rapport à l'activité pure de l'intelligence abstraite, de même que la sensation sera définie comme différentielle d'activité. Par suite, l'aporie sur laquelle débouchent les «quelques leibniziens " se laisse formuler simplement : l'idéalisme ne peut expliquer la représentation (la direction du moi vers un X qui n'est pas le moi). Si les leibniziens avaient raison, l'accident (par exemple la sensation) devrait être perçu comme une modification du moi, et non être référé à un objet extérieur. Pourquoi le moi exclut-il cette modification en la rapportant à un monde, alors que, selon l'explication du philosophe leibnizien, il devrait percevoir le monde comme une illusion ou une production construite à partir de l'intelligence ? En bref, écrit Fichte si les Leibniziens avaient raison "le moi devrait se considérer comme étant la cause de cette limitation $»^{24}$. Ce que l'idéalisme ne peut expliquer c'est donc la représentation comme relation à un autre.

\section{II.2. Le réalisme et l'idéalisme quantitatif.}

Envisageons maintenant le réalisme et l'idéalisme quantitatif, qui sont analysés beaucoup plus tard dans la Grundlage ${ }^{25}$. Si l'idéalisme et le réalisme qualitatif renvoyaient à des systèmes du XVIIe siècle, l'idéalisme et le réalisme quantitatifs renverront à des systèmes kantiens : Kant est ainsi explicitement cité dans le camp des "réalistes quantitatifs»; quant à l'idéalisme quantitatif, il est possiblement incarné par Maimon ${ }^{26}$, même si Fichte ne donne ici aucun nom. La question qui se pose d'abord à nous, avant toute analyse du contenu de ces positions, est de savoir quelle est la différence entre le qualitatif et le quantitatif?

Fichte nous dit qu'il s'agit d'une différence d'abstraction, mais on peut aussi montrer qu'il s'agit de ce que l'on pourrait nommer une différence « d'internalisation ». En effet, il faut se souvenir qu'avec Descartes et la philosophie du XVIIe, le problème de la représentation se posait en termes de confrontation d'un sujet subsistant et d'un objet existant en dehors de lui. Il s'agissait de mettre en relation ces deux entités extérieures l'une à l'autre. Le propre de la révolution copernicienne est fondamentalement de penser le rapport entre l'objet et le sujet à l'intérieur même de la représentation. Elle consiste à ne plus considérer « sujet » et « objet » comme deux termes extérieurs mais 
comme des vecteurs internes à la représentation (voir, par exemple, le premier principe de Reinhold). Parce que l'objet, dont la Critique s'occupe, est phénomène et non plus chose en soi, Kant peut passer d'une problématique de l'adéquation entre sujet et objet, propre au XVIIe siècle, à une problématique de la validité objective. Avec Kant et son intériorisation de l'objet, la relation sujet/objet devient la relation entre des représentations singulières et des concepts généraux, soit la relation entre intuition et entendement. La différence entre quantitatif et qualitatif est donc d'abord à imputer à ce changement de perspective, c'est-à-dire à cette internalisation de la relation, que la révolution copernicienne a opérée.

Parallèlement, le niveau qualitatif restait polarisé sur la seule relation sujet-objet et ne thématisait pas frontalement la composition entre relation acte/passivité et activité indépendante. Or, et c'est ce qui est décisif, le réalisme et l'idéalisme quantitatif prennent en compte l'activité indépendante et la nécessité de la composer avec la relation acte-passivité ; pour le dire autrement, ils tentent de penser à la fois la représentation et la réflexion. Le problème est que la composition qu'ils en effectuent est aporétique et requiert donc d'être dépassée. Envisageons plus précisément ce point à partir de Kant, que Fichte définit comme « réaliste quantitatif ».

Il convient de remarquer, au préalable, que face à cette assignation de Kant dans le camp du « réalisme » le lecteur est en droit de manifester sa surprise : pourquoi Kant se retrouve du côté des réalistes? Comment Fichte peut-il faire de Kant un réaliste, alors même qu'il affirme que Kant reste à l'intérieur du sujet (internalisation de la relation) ?

Cette déconcertante assignation nous autorise une remarque qui sera décisive pour comprendre, in fine, ce que Fichte entendra par «idéalisme achevé ». Une lecture trop rapide de certains textes plus populaires de Fichte a pu faire penser à une bonne partie du commentarisme que les réalistes partaient de l'objet et les idéalistes, du sujet. L'idéaliste, dans ces différentes variantes, resterait à l'intérieur du sujet et tenterait d'en déduire l'altérité. Mais pourtant, on le voit très clairement ici, Kant est dit " réaliste ", alors même qu'il se tient à l'intérieur du sujet, puisque la différence entre le réalisme qualitatif et quantitatif est une différence d'internalisation: le réaliste qualitatif sort du sujet pour postuler une cause extérieure en soi (Dieu ou la nature), le réaliste quantitatif reste à l'intérieur du sujet. Il est clair, à la lumière de ces analyses, que l'équation trop courante selon laquelle idéaliste $=$ sujet et réalisme $=$ objet est fausse. L'idéaliste ne signifie pas, pour Fichte, celui ne sort pas du sujet, puisque, dans cette typologie de la Grundlage, Kant est dit réaliste, alors même que, Fichte y insiste ${ }^{27}$, Kant ne sort pas du sujet pour postuler un objet en soi.

Mais alors, derechef, pourquoi réaliste? Quelle est la nature de l'erreur de Kant selon la Grundlage? Essayons de comprendre ce que Fichte reproche à Kant ici, ce qui nous permettra d'approcher sa propre signification de l'idéalisme.

En fait, le réalisme quantitatif ne postule aucune extériorité réelle, il ne déduit pas la scission sujet/objet, il la constate : «il affirme l'existence réelle d'une limitation du moi, indépendamment d'un acte du moi ", nous dit Fichte. Ce que Kant admet n'est pas une chose en soi (comme Spinoza) mais une finitude "en soi ». La passivité est la facticité de départ, ce que l'on trouve, ce qui est "donné », mais que le philosophe ne peut expliquer. Pour Kant, la finitude est indéductible. C'est, nous dit Fichte : « une finitude contingente ». Alors que le réalisme qualitatif tente de déduire la finitude à partir de son autre (Dieu ou la nature comme chose en soi), le réalisme quantitatif n'exprime rien d'autre que l'impossibilité à déduire la finitude. Reconnaissance de la 
facticité ou de la « finitude contingente », que beaucoup, depuis Heidegger, portent au crédit de Kant, mais qui aux yeux de Fichte pose la question suivante « Comment Kant sait-il que nous sommes finis?».

31 Pour ne pas mésinterpréter la critique de Fichte ici, en considérant sa demande d'une « déduction de la finitude » comme symptôme d'une sortie hors des limites de la pensée transcendantale, il convient de rappeler que « déduire » pour Fichte signifie démontrer la légitimité d'une assertion. La demande de Fichte est donc : « quel est le point de vue à partir duquel Kant peut dire que l'homme est fini?». Si ce point de vue ne peut être explicité, si ce qui est dit ne peut avoir d'autre statut que celui d'un « en soi » constaté, alors nous n'avons aucune raison philosophique de l'admettre. En effet, le philosophe en disant que « l'homme est fini » prétend à la vérité de cette proposition. Mais si cette prétention ne peut être justifiée (déduite au sens du quid juris), alors il s'agit d'un dogme indémontrable ou d'une proposition admise pour des raisons extraphilosophiques. C'est pourquoi, face à la finitude, Kant se trouve dans la même position que le réaliste qualitatif face à l'objet en soi. Il devient ainsi un réaliste et plus précisément un réaliste du fini. Fichte estime donc que, par-delà les apparences, réalisme qualitatif (Spinoza) et réalisme quantitatif (Kant) "commettent la même faute » et il insiste «je dis bien: en commettant la même faute : en effet il lui est (à Kant) impossible d'expliquer comment une détermination réelle peut devenir idéale ${ }^{28}$. Certes, le réalisme kantien, ne doit pas être critiqué à partir de ce qu'il dit du rapport de l'objet au sujet représentant (problématique de la représentation), mais à partir de ce que le philosophe dit du sujet de la représentation, c'est-à-dire à partir de ce que le sujet dit de lui-même, ou de ce que le philosophe dit de l'homme (par exemple qu' " il est fini »). Si nous analysons la position de Kant sur la finitude, nous nous apercevons qu'il reproduit, à un autre niveau, qui est celui de la réflexion, le geste du réaliste qualitatif. Par suite, dira Fichte: «on ne voit pas pourquoi nous devrions accorder notre croyance à l'affirmation indémontrée de l'un plutôt qu'à l'affirmation indémontrée de l'autre ».

\section{3. Quatre enseignements doivent être tirés de cette curieuse assignation de Kant dans le camp des « réalistes ».}

1) Kant n'est pas dit réaliste parce qu'il sortirait du sujet pour partir d'un objet. La différence entre réaliste et idéaliste ne passe donc pas par cet axe, pourtant rebattu par la suite.

2) Le reproche fait à Kant est très précisément de ne pas pouvoir « expliquer comment une détermination réelle peut devenir idéale ». Il ne peut être dit «idéaliste » parce que, sur ce point, il n'accède pas à l'idéalité. La définition de l'idéalisme est donc liée à la notion "d'idéalité" plus qu'à celle de subjectivité. Ce que corroborera l'analyse ultérieure du dernier niveau du réalisme, le réalisme «abstrait $»^{29}$, qui représente encore un pas supplémentaire dans l'internalisation, puisque nous dit Fichte, pour ce réaliste abstrait: "l'objectif qui doit être exclu n'a nul besoin d'exister " ${ }^{30}$. Il n'en sera pas moins dit « réaliste » et récusé comme Kant l'a été. Parallèlement, l'idéaliste abstrait qui prétend que: "l'intelligence avec toutes ses déterminations possibles serait le résultat de la seule spontanéité $»^{31}$, et semble, dès lors, incarner la position du sujet absolu à partir duquel le monde surgit, sera dit insuffisamment «idéaliste » par Fichte. Mais cela, non pas parce que l'on pourrait atteindre un niveau encore plus 
" subjectiviste ", mais bel et bien parce que l'idéaliste abstrait échoue à rendre compte de cette proposition, qui doit donc être récusée par l'idéalisme achevé. Ce dernier niveau, à savoir celui du réalisme et de l'idéalisme abstrait, confirment donc nos analyses : l'idéalisme, pour Fichte, n'est pas lié à la notion de subjectivité mais à celle d'idéalité.

3) Cette idéalité consiste pour le philosophe, ou sujet de la réflexion, à pouvoir rendre compte de la possibilité de sa propre énonciation. Il s'agit de faire accéder nos énoncés au savoir philosophique et donc scientifique, et non d'en rester à des constats contingents ou facticiels (faktisch), dont on ne peut établir la validité et qui se révèlent, in fine, tous auto-contradictoires. L'idéalité renvoie donc à la notion de validité. Puis je ou non justifier la validité de tel ou tel énoncé ? Telle est la question. Par exemple, dans le cas de Kant: peut-il justifier sa prétention à la validité contenu dans l'énoncé «l'homme est fini »?

4) Dans la proposition précédemment citée, selon laquelle Kant est réaliste car « il lui est impossible d'expliquer comment une détermination réelle peut devenir idéale ", il ne s'agit pas pour Fichte de nier la " détermination réelle ", c'est-à-dire de contester l'effectivité ou l'existence de tel ou tel X, mais de dire que ce qui est posé comme réel (dans le cas présent, la finitude) doit devenir, en philosophie, c'est-à-dire du point de vue de la réflexion, "idéale ", c'est-à-dire doit faire l'objet d'une démonstration (déduction) et non d'un simple constat empirique ou «contingent». La question de la validité du sens est donc celle de la possibilité, pour la philosophie, de justifier ses énoncés. L'idéalisme authentique sera celui qui peut articuler, sans auto-contradiction, le contenu de ses énoncés et la possibilité de leur énonciation ${ }^{32}$. On comprend dès lors pourquoi Fichte, comme Hegel, dira qu'il n'est de philosophie qu'idéaliste ${ }^{33}$. Ou bien nous avons des constats contingents que rien ne justifie («des affirmations indémontrées»), ou bien nous pouvons honorer notre prétention à la vérité et ainsi entrer dans la sphère du savoir et de la doctrine de la science. Savoir qui ne consiste pas seulement à penser, décrire ou dire, mais bel et bien à démontrer comment l'on peut dire, décrire et penser. Cette exigence de noesis noeseos, qui conclut l'Encyclopédie de Hegel, est déjà pour Fichte la signature de l'idéaliste.

Parce que l'idéalisme a affaire à la validité du sens, sa tâche consiste à faire accéder à l'idéalité toutes les dimensions que l'on dira "réelles ». Plus exactement pourront être dites réelles les dimensions dont on peut montrer qu'elles sont idéales, c'est-à-dire justifiées, démontrables sans auto-contradiction. La destination de la philosophie n'est donc pas de dire seulement ce qui est (réalité), mais de montrer par quels actes ou procédures le philosophe peut dire ce qui est (idéalité); soit pour reprendre la proposition par laquelle Fichte apostrophe souvent ses prédécesseurs, et particulièrement Kant : «Comment le sait-il et d'où le sait-il ?» (Id est : «Comment le philosophe peut-il dire ce qu'il dit »)

Au terme de ces analyses, nous accédons à la définition de «l'idéalisme achevé » qui se confond, chez Fichte, avec la philosophie même ou doctrine de la science. L'idéalisme achevé pourra rendre compte de l'intégralité des énoncés qu'il avance, les déduire c'est-à-dire en montrer la légitimité, en justifier le statut et la possibilité. Démontrer un énoncé ne signifie nullement produire, à partir du sujet, et à la manière d'un démiurge, la totalité du réel ou de l'existence. Démontrer l'intégralité des énoncés proposés (par exemple la proposition selon laquelle «l'homme est fini ») est une question relative à la validité du sens, à la vérité d'une proposition. Cette analyse de la Grundlage nous dévoile 
que l'idéalisme est intrinsèquement lié à la notion d'idéalité et de validité, et non de subjectivité.

Cette démonstration faite, je voudrai, comme annoncé, conclure sur une note plus contemporaine en m'interrogeant sur le curieux destin du concept d'idéalisme aujourd'hui, c'est-à-dire ces trente dernières années.

\section{Conclusion}

Les philosophes qui, aujourd'hui, revendiquent explicitement le terme « idéaliste » sont rares, voire inexistants. L'adjectif ne semble plus devoir désigner que des penseurs du passé : les représentants de l'idéalisme allemand, puis, au début du vingtième siècle, Bradley et Husserl. Mais si l'idéalisme n'est plus guère revendiqué aujourd'hui, cela ne signifie pas qu'il ait disparu des livres nouvellement écrits, loin s'en faut. En effet, depuis une trentaine d'années, le terme est abondamment utilisé par la pléthore de philosophes qui se réclament du «réalisme», lequel représente la "constellation conceptuelle » majeure de notre début de vingt-unième siècle, comme l'idéalisme avait incarné celle du début du dix-neuvième. Or les réalistes contemporains utilisent le terme "idéalisme » comme un antonyme du réalisme, un ennemi à abattre, voire un épouvantail. Il n'est pour s'en convaincre que de citer Bouveresse qui définit le réalisme comme «se méfier systématiquement de l'idéalisme ${ }^{34}$, Tiercelin qui parle de la «puissante menace idéaliste qui pèse aujourd'hui, y compris sur la science et en particulier la physique $\|^{35}$, ou encore Meillassoux qui voit, dans l'idéalisme kantien et ses multiples variantes, une "catastrophe " $^{36}$. On peut, de même, relever, du côté de la phénoménologie la plus contemporaine, la reprise quasi obsessionnelle d'un même titre, celui de « révolution anti-copernicienne » (Richir, Romano, Pradelle); révolution anti-copernicienne qu'il est aisé de comparer à la révolution ptolémaïque, souhaitée par Meillassoux dans Après la Finitude. Bref, une grande partie de la philosophie de ces trente dernières années a remis sur le devant de la scène la querelle de l'idéalisme et du réalisme, mais de manière éminemment réactive puisque l'idéalisme ne sert plus au réalisme qu'à se poser en s'opposant, à se déterminer en rejetant, à s'identifier en excluant. Or, de ces occurrences, purement négatives, du terme «idéalisme » émerge une signification centrale, à savoir que l'idéaliste serait celui qui, niant la réalité du monde extérieur, professe un subjectivisme débridé, qui, immanquablement, débouche sur un relativisme généralisé. Evoquons, pour le montrer, un exemple, parmi tant d'autres, à savoir la manière dont Tiercelin entend réfuter l'idéalisme hégélien. Dans son petit traité de métaphysique scientifique réaliste de 2011, elle n'hésite pas à écrire contre Hegel : « un coup de poing viendra à bout de tous les arguments idéalistes niant la réalité du monde extérieur $\rrbracket^{37}$, montrant par-là que l'idéalisme est, à ses yeux, une sorte de solipsisme radical, pour lequel les représentations de l'individu sont seules tangibles, et pour lequel, ce faisant, un monde extérieur n'existe pas. Or, comme le soulignait déjà $\mathrm{Hegel}^{38}$, aucun philosophe idéaliste n'a jamais, dans l'histoire, soutenu ce type de position; ce que nous avons amplement vérifié ici avec Fichte lui-même. Ce contresens sur la nature de l'idéalisme se retrouve un peu partout dans la littérature contemporaine et la recension de ses multiples occurrences ne ferait que démontrer l'impressionnante persistance de cette association entre "idéalisme » et subjectivisme ou relativisme. Comment comprendre ce que je caractériserai comme le contresens le plus spectaculaire et en même temps le plus vivace de toute l'histoire de la 
philosophie ? Je ne puis, dans le cadre limité de cet exposé, retracer l'histoire de ce contresens qui commence chez Diderot, s'amplifie avec Moore et traverse toutes les pensées dites du sens commun ou Popular philosophie ${ }^{39}$. Néanmoins, je puis au terme de ce parcours en suggérer le ressort. Cette source, à mon sens, se trouve dans un geste contemporain central qui consiste à rabattre la question de la vérité ou de la validité du sens sur la seule réalité de la chose ou de l'expérience. L'expérience (comme "donné » chez les phénoménologues, comme « usage » chez les wittgensteiniens ou comme ce qui est matériellement là -«le grand dehors »- chez les métaphysiciens contemporains) vaut du fait de sa seule occurrence, qu'il suffit d'exhiber. La réalité prime et a recouvert la question de la vérité. Il ne s'agit plus de s'enquérir, dans la philosophie d'aujourd'hui, de ce qui est vrai mais de montrer ce qui est réel. Pour le dire, à partir de Fichte, ce qui est occulté est la deuxième série de son analyse, à savoir celle de l'activité indépendante, qui introduit à la question de la consistance de l'énonciation du philosophe. Le réalisme contemporain, toutes tendances confondues, tend à occulter cette distance réflexive et à s'enfermer ainsi dans des contradictions performatives sans fin ${ }^{40}$ où, comme chez Spinoza selon Fichte, le contenu de l'énoncé est invalidé par l'acte même de son énonciation. Le contresens contemporain sur l'idéalisme consiste donc bien d'une part à rattacher le concept d'idéalisme à la notion de subjectivité quand, comme nous l'avons vu, il se rattache, depuis sa naissance, à la notion d'idéalité, et d'autre part à le penser à partir de la catégorie de réalité ou d'existence de la chose, là où il était d'abord question, pour l'idéalisme, de la validité du sens. La plongée dans le labyrinthe de l'idéalisme nous permet de dénoncer ce contresens et de présenter ainsi sous un autre angle la querelle de l'idéalisme et du réalisme qui traverse et anime encore la philosophie de notre XXIe siècle.

\section{NOTES}

1. HEGEL, G. W.F., Werke in 20 Bänden, Frankurt a.M.: Surhkamp, 1986, Bd. 8, p. 203, et Bd. 5, p. 172: «l'opposition de la philosophie idéaliste et réaliste est dépourvue de signification. Une philosophie qui attribuerait à l'être là fini comme tel un être véritable, ultime, absolu ne serait pas digne du nom de philosophie ».

2. GWL, GA I/2, 300, traduction française par A. Philonenko, FICHTE, J. G, Principes de la doctrine de la science, in: CEuvres choisies de philosophie première. Doctrine de la Science (1794-1797), (=OCPP), Paris : Vrin, 1980, p. 53.

3. LEIBNIZ, G. W., Système de la nature et de la communication des substances, Paris : Flammarion, 1994, p. 198. Leibniz définit les idéalistes comme «les adeptes de la philosophie des idées", qui accordent réalité et primauté aux Idées du monde supra-sensible et les oppose aux matérialistes (Epicure). Sur cette histoire de l'idéalisme avant Kant, voir mon article «Les idéalistes avant l'idéalisme ».

4. WOLFF, Ch., «Préface » (datée de 1719) des Vernunftige Gedanken, Halle 1720. Dans la typologie wollfienne, la signification pertinente de l'idéalisme demeure l'affirmation de la primauté et de la réalité du monde de l'esprit par opposition aux matérialistes qui ne reconnaissent d'autre règne que celui de la matière (Epicure) ou de la nature comprise selon les lois du mécanisme (Hobbes). 
Le seul exemple donné d'idéaliste, outre l'évocation d'un évanescent «disciple parisien de Malebranche ", est Berkeley.

5. DIDEROT, D., Lettre sur les aveugles. Londres, 1749. Sur l'importance de Diderot dans la progressive définition (réactive) de l'idéalisme, voir notre article, précédemment cité : «Les idéalistes avant l'idéalisme ».

6. La recension paraît dans Annonces savantes de Gottingen du 19 janvier 1782. C'est cette recension qui est à l'origine de la transformation la plus substantielle de la seconde édition, selon l'aveu même de Kant. La critique de Jacobi, parue simplement quelques mois avant la publication de la seconde édition ne fait pas l'objet, de la part de Kant, d'une réponse circonstanciée. Même si Kant revient en 1790 sur dans ses Reflexionen sur la question de l'idéalisme, il semble néanmoins que pour lui, la question est réglée par la seconde édition. Cette critique de Jacobi beaucoup plus élaborée et percutante que celle des Annonces (dont Kant n'hésite pas à dire qu'elle constitue un contresens massif sur son entreprise) aura plus d'importance pour les successeurs immédiats de Kant. La recension des Annonces est l'œuvre, involontairement collective, de Garve et Feder qui tous deux appartiennent à la philosophie du Common sense, devenue Popular Philosophie en Allemagne, courant dont l'influence est considérable du temps de Kant. Kant s'y fait accuser « d'idéalisme transcendant » et est sommé de montrer en quoi il diffère de Berkeley.

7. Voir aussi son important Appendice qui prend directement à parti cette recension.

8. KrV A 39, A 367ss., A 490-497.

9. $\mathrm{EE}, \mathrm{GA} \mathrm{I} / 4,200-201$. Selon l'expression qu'il emploie dans l'Essai d'une nouvelle présentation de la doctrine de la science, 1797/98, trad. fr. I.Thomas-Fogiel, Paris : Vrin 1999 (à l'avenir NPDS).

10. . Voir dans le même sens la lettre à Reinhardt (GA III/2, 39). Il ajoute : "Mais il a renforcé en moi cette conviction qu'elle pourra le devenir en peu de temps."

11. Fichte écrit : "Envers le talent de Maïmon, mon admiration est sans limites. Je crois et je suis prêt à le prouver qu'il a bouleversé de fond en comble toute la philosophie kantienne". Sur l'influence de Maimon sur Fichte, voir mon livre Critique de la représentation, Etude sur Fichte, Paris: Vrin, 2000.

12. Cette alternative, exprimée sous de multiples formes dans l'Enésidème, sera magistralement résumée par l'auteur dans un de ses autres ouvrages : Critique de la philosophie théorétique: “Donc si ce que la Critique affirme savoir des fondements de l'expérience est une connaissance réelle, l'affirmation de la même Critique, selon laquelle toute connaissance vraie de notre esprit est limitée exclusivement aux objets de l'expérience, est absolument fausse. Si, par contre, cette affirmation devait être vraie, toute connaissance des sources de l'expérience doit être tenue dans son ensemble comme une apparence vide Aetas kantiana, t. 244, en deux volumes, vol. II, Bruxelles, 1968, p.578. Sur cette critique d'Enésidème, voir mon livre: Fichte, réflexion et argumentation, Paris : Vrin 2004.

13. Nature comprise de manière scientifique comme ensemble de régularités, et non pas de manière sensible et ordinaire comme environnement dans lequel l'individu est plongé.

14. Voir sa réponse à la recension Garve-Feder : "La thèse de tous les idéalistes authentiques depuis les éléates jusqu'à Berkeley est contenue dans cette formule : toute connaissance par les sens et l'expérience n'est qu'une simple apparence et c'est seulement dans les idées de la raison et de l'entendement pur qu'il y a de la vérité » (Prol., AA 04, 374, trad. fr. KANT, I., CEuvres philosophique, 3 vol., dir. F. Alquié, , Paris: Gallimard, 1980, t. 2, p. 160. Pour Kant, ce qui est pertinent pour définir l'idéalisme est la notion d'idées ou d'idéalités.

15. On la retrouvera clairement thématisée par Husserl dans les Méditations Cartésiennes (comme moi phénoménologisant et moi constituant), sur ce point voir mon ouvrage : Le lieu de l'universel, impasses du réalisme dans la philosophie contemporaine, Paris : Seuil, 2015.

16. Cf. GWL, GA I/2, 306 (OCPP 59) où la " contradiction » et la nécessité de composer les deux séries est exposée « de façon précise » selon les termes de Fichte (GA I/2, 304/OCPP 56). Soit : 1) 
«Par la relation acte-passivité, une activité indépendante est déterminée ». 2) Par une activité indépendante, une relation acte-passivité est déterminée. 3) Ces deux propositions se déterminent mutuellement... ».

17. Dans NPDS Fichte détermine l'idéalisme de Kant comme « inachevé « et lui oppose l'idéalisme « achevé » de la doctrine de la science. Il écrit aussi «il ne reste plus comme seule philosophie possible que l'idéalisme », vide supra n. 9.

18. GWL, GA I/2, 307-384 (OCPP 59-121).

19. Dans la Grundlage (GA I/2,333/OCPP 80), Fichte dit ainsi que l'idéalisme quantitatif est «plus abstrait» que l'idéalisme qualitatif, et que l'idéalisme abstrait représente encore un pas supplémentaire d'abstraction par rapport à l'idéalisme quantitatif.

20. GWL, GA I/2, 302-304 (OCPP 54-56).

21. La preuve en est que la discussion autour de ce réalisme et idéalisme qualitatif intervient juste avant la formulation de l'activité indépendante. En effet, l'activité indépendante apparaît (GA I/2, 305/OCPP 57: "Nous nommerons cette sorte d'activité, pour le moment, l'activité indépendante, en attendant de mieux la connaître »), alors que le cercle de l'idéalisme et réalisme qualitatif est discuté (GA I/2, 302-304/OCPP 54, ligne 41 à 56, ligne 38). C'est en fait ce cercle qui produit la découverte de l'activité indépendante. Précisons que Fichte ne désignera cet idéalisme et réalisme comme « qualitatif » que plus tard dans la Grundlage, lorsque précisément il parlera d'une forme supérieure car "plus abstraite » de l'idéalisme (GA I/2, 333/OCPP 80), à savoir l'idéalisme quantitatif, «pour les distinguer, je nommerai ce premier idéalisme, qui supprime quelque chose de posé en soi, l'idéalisme qualitatif; et le second, qui pose originairement une quantité limitée, l'idéalisme quantitatif » (GA I/2, 185/OCPP 81).

22. GWL, I/2, 303 (OCPP 55-56).

23. Sur le rôle central de la contradiction performative chez Fichte voir mon livre Critique de la représentation, étude sur Fichte, Paris : Vrin 2000 et mon commentaire dans NPDS, pp. 36-42.

24. GWL, GA I/2, 303-304 (OCPP 56).

25. GWL, GA I/2, 330-335 (OCPP 79 à 82).

26. Sur cette hypothèse et sa justification voir mon livre Critique de la représentation, p. 174.

27. Fichte écrit «certes le réalisme quantitatif doit, étant donné le principe qu'il développe, rapporter cette limitation au Non-Moi comme à sa raison réelle; mais il sait que ce principe n'est que pour lui, et ce faisant, il n'est victime d'aucune illusion » (GWL, GA I/2, 335/OCPP 82). Disant cela, il montre que pour lui, Kant ne pose pas de « chose en soi » comme réalité, cause de nos représentations (objection de Jacobi contre Kant). Il n'est ainsi victime « d'aucune illusion ».

28. GWL, GA I/2, 336/OCPP 82).

29. Fichte écrit à propos de ce réalisme « Il est clair que ce réalisme est beaucoup plus abstrait que les réalismes précédemment définis » (GA I/2, 355/OCPP 97) et établit la différence précise entre réalisme quantitatif (Kant) et ce réalisme abstrait: " pour le réalisme quantitatif, la détermination était donnée, maintenant elle doit être produite»(ibid). Ce réalisme échoue également « il commet la faute propre à tout réalisme » (GA I/2, 355/OCPP 98).

30. C'est à ce moment qu'apparaît le fameux «choc» (Anstoss). La première occurrence de la notion se trouve donc au sein d'une position (le réalisme abstrait) qui est réfutée par Fichte (GWL, GA I/2, 355). Ce qu'il ne faut pas perdre de vue si l'on veut comprendre la véritable signification de l'Anstoss.

31. GWL, GA I/2, 354 (OCPP 96).

32. Il s'agit de la congruence entre le Tun (faire) et le Sagen (dire), congruence partout réclamée par Fichte, et qui, à mon sens, est l'axe central de sa philosophie. Sur ce point voir mes livres (2000 et 2004), où je présente la philosophie de Fichte comme une logique des actes ou grammaire de l'effectuation: «La philosophie devient bien une logique des actes, une actologie destinée à 
remplacer l'antique ontologie » (Fichte, réflexion et argumentation, p.178), ainsi que mes préfaces aux traductions françaises des $W L(98,1805,1812)$ de Fichte, par exemple in livre de poche, 2000 : «à l'ontologie traditionnelle (qu'est-ce que l'être ?) Fichte substitue une actologie (qu'est-ce que l'acte ?)» (Critique de la représentation, p. 21).

33. «Il ne reste plus comme unique philosophie possible que l'idéalisme » (EE, GA I/4, 198/NPDS 111).

34. BOUVERESSE, J., Le Philosophe et le Réel, Paris : Hachette Littératures, 1998, p.35.

35. TIERCELIN, C., Le ciment des choses. Petit traité de métaphysique scientifique réaliste, Paris: Les Éditions d'Ithaque, 2011 p. 28.

36. MEILlassouX, Q., Après la finitude, Paris : Seuil 2004.

37. TIERCELIN, C., Le ciment des choses, p. 226.

38. «L'idéalisme, dit-on souvent, consiste à affirmer que l'individu engendre par lui-même toutes ses représentations, même les plus immédiates, qu'il pose tout à partir de lui-même. C'est là toutefois une représentation non-historique et fausse. Si l'idéalisme est tel que ce grossier mode de représentation le définit, il n'y a en fait jamais eu aucun idéaliste parmi les philosophes » (Leçons sur Philosophie de l'histoire, Paris, Vrin, 1964, t. 3, p.427)

39. Pour de plus amples détails sur cette histoire, je renvoie à mon article déjà cité « les idéalistes avant l'idéalisme " et à un ouvrage à paraître, qui analyse ce curieux destin du concept «d'idéalisme » et détaille l'origine du contresens (la philosophie du sens commun, dite Popular Philosophie en Allemagne, puissant mouvement auquel appartiennent, et ce n'est pas un hasard, Garve et Feder.)

40. J'en ai établi la liste dans mon dernier livre Le lieu de l'universel, impasses du réalisme dans la philosophie contemporaine, Seuil 2015.

\section{RÉSUMÉS}

Why was Kantian idealism so inadequate in Fichte's eyes that he needed to re-found the whole of philosophy in his Grundlage, based on a thorough re-examination of the intricacies of idealism leading to its ultimate re-definition?

In order to answer this question, I will first examine the original question of the «Science of knowledge » (94/95). I will then analyze the meaning of how Fichte, in the Grundlage, typologizes various realisms and idealisms as being either qualitative, quantitative or abstract. This will allow me to show that Fichtean idealism is not determined by any kind of " philosophy of the subject " but rather by the question of the ideality of statement as raised by the problem of the nature of the enunciation of philosophic speech. As a conclusion, I will show how contemporary realism, which is characteristically critical of "idealism ", is profoundly mistaken on two fundamental questions: firstly, because it establishes a link between idealism and subjectivity whereas it has been linked to ideality; secondly, because it defines idealism in the light of the categories of reality and of the existence of the thing whereas it is above all about the question of how one ascertains meaning. 
INDEX

Keywords : idealism, realism, skepticism, transcendental, speculative realism

AUTEUR

ISABELLE THOMAS-FOGIEL

Université d'Ottawa/Université de Paris 1, Panthéon-Sorbonne 